\title{
Charge Frustration Effects in Capacitively Coupled Two-Dimensional Josephson-Junction Arrays
}

\author{
Minchul Lee, Mahn-Soo Choi*, and M. Y. Choi \\ Department of Physics and Center for Theoretical Physics, Seoul National University, Seoul 151-742, Korea
}

\begin{abstract}
We investigate the quantum phase transitions in two capacitively coupled two-dimensional Josephson-junction arrays with charge frustration. The system is mapped onto the $S=1$ and $S=1 / 2$ anisotropic Heisenberg antiferromagnets near the particle-hole symmetry line and near the maximal-frustration line, respectively, which are in turn argued to be effectively described by a single quantum phase model. Based on the resulting model, it is suggested that near the maximal frustration line the system may undergo a quantum phase transition from the charge-density wave to the super-solid phase, which displays both diagonal and off-diagonal long-range order.
\end{abstract}

PACS numbers: 74.50.+r, 67.40.Db, 73.23.Hk

In recent years, various types of cotunneling transport have been of great interest in ultrasmall tunnel junc tions, which exhibit strong Coulomb blockade effect.t In particular, cotunneling of the electron-hole pairs in two capacitively coupled one-dimensional (1D) arrays of small metallic junctions has been proposed theoretically 2 and demonstrated experimentally 3 revealing the remarkable effects of the curfent mirror. More refently, in capacitively coupled $1 \mathrm{D}$ 组 or two-dimensional $(2 \mathrm{D})$ Josephson-junction arrays (JJAs), the cotunneling of particle-hole pairs (with the particle and hole standing for the excess and deficit Cooper pair, respectively) has been proposed even to drive the quantum phase transition from superconductor to insulator (SI) at zero temperature. Here the particle-hole symmetry of the system may be broken by, e.g., the gate voltage applied between the array and the substrate. The resulting charge frustration is expected to affect the phase transition of the system in a crucial way. For example, when the particle-hole symmetry is broken maximally, the transport is governed by the cotunneling of the particle-void pairs (with the void denoting the absence of an excess or deficit Cooper pair) and the different nature of the associated phase transition has been pointed out in one dimension. 0 On the other hand, existing studies of coupled 2D arrays with charge frustration have concentrated upon the charge-vortex duality, 0 without appreciable attention to the phase transitions.

In this paper, we extend the previous work 6 on two capacitively coupled 2D arrays of ultrasmall Josephson junctions to investigate the charge-frustration effects on the quantum phase transitions. In a manner similar to that of Ref. A, we map the system to the $S=1$ anisotropic Heisenberg antiferromagnet near the particlehole symmetry lines and to the $S=1 / 2$ one near the maximal-frustration lines. It is then argued that the two spin models can in effect be incorporated into a single $2 \mathrm{D}$ quantum phase model with the effective self-capacitance given by the coupling capacitance of the original twoarray system and the junction capacitance by the intraarray junction capacitance. The resulting model indicates that near the maximal frustration line the system may exhibit a quantum phase transition from the charge- density wave (CDW) to the super-solid (SS) phase. In the SS state, the system possesses both diagonal and offdiagonal long-range order (DLRO and ODLRO): Namely, both the density-correlation of charges and the phasecorrelation of superconducting order parameters remain finite as the distance grows arbitrarily large.

The system of coupled 2D square arrays, shown schematically in Fig. 1, is described by the Hamiltonian

$$
\begin{aligned}
H= & 2 e^{2} \sum_{\ell, \ell^{\prime} ; \mathbf{r}, \mathbf{r}^{\prime}}\left[n_{\ell}(\mathbf{r})-n_{g}\right] \mathbb{C}_{\ell \ell^{\prime}}^{-1}\left(\mathbf{r}, \mathbf{r}^{\prime}\right)\left[n_{\ell^{\prime}}\left(\mathbf{r}^{\prime}\right)-n_{g}\right] \\
& -E_{J} \sum_{\ell, \mathbf{r}, \mu} \cos \left[\phi_{\ell}(\mathbf{r})-\phi_{\ell}\left(\mathbf{r}+\hat{\mathbf{e}}_{\mu}\right)\right] \\
\equiv & H_{C}+H_{J}
\end{aligned}
$$

where the number $n_{\ell}(\mathbf{r})$ of the Cooper pairs and the phase $\phi_{\ell}(\mathbf{r})$ of the superconducting order parameter at site $\mathbf{r}$ on the $\ell$ th array $(\ell=1,2)$ are quantummechanically conjugate variables: $\left[n_{\ell}(\mathbf{r}), \phi_{\ell}\left(\mathbf{r}^{\prime}\right)\right]=$ $i \delta_{\ell \ell^{\prime}} \delta_{\mathbf{r r}^{\prime}}$. The Josephson coupling between neighboring sites $\mathbf{r}$ and $\mathbf{r}+\hat{\mathbf{e}}_{\mu}$ (with $\hat{\mathbf{e}}_{\mu}$ being the unit vector in the direction $\mu=x, y$ ) in each array is characterized by the coupling energy $E_{J}$, whereas the external charge $n_{g} \equiv C_{0} V_{g} / 2 e$ induced on each island by the applied gate voltage $V_{g}$ breaks the particle-hole symmetry of the system, introducing charge frustration. The two arrays are coupled through the capacitance $C_{I}$ between two grains at the same position $\mathbf{r}$ on the two arrays. (Note the differ ence from the Josephson coupled multi-layered system, where Cooper-pair tunneling between layers is allowed.) The capacitance matrix $\mathbb{C}$ characterizing the charging energy part $H_{C}$ of the Hamiltonian in Eq. (11) can be written in the block form:

$$
\mathbb{C}_{\ell \ell^{\prime}}\left(\mathbf{r}, \mathbf{r}^{\prime}\right) \equiv C\left(\mathbf{r}, \mathbf{r}^{\prime}\right)\left[\begin{array}{ll}
1 & 0 \\
0 & 1
\end{array}\right]+\delta_{\mathbf{r}, \mathbf{r}^{\prime}} C_{I}\left[\begin{array}{rr}
1 & -1 \\
-1 & 1
\end{array}\right],
$$

where $C\left(\mathbf{r}, \mathbf{r}^{\prime}\right)$ is the usual intra-array capacitance matrix

$$
\begin{aligned}
C\left(\mathbf{r}, \mathbf{r}^{\prime}\right) & \equiv C_{0} \delta_{\mathbf{r} \mathbf{r}^{\prime}} \\
& +C_{1} \sum_{\mu}\left[2 \delta_{\mathbf{r r ^ { \prime }}}-\delta_{\mathbf{r}, \mathbf{r}^{\prime}+\hat{\mathbf{e}}_{\mu}}-\delta_{\mathbf{r}, \mathbf{r}^{\prime}+\hat{\mathbf{e}}_{\mu}}\right] .
\end{aligned}
$$


with $C_{0}$ and $C_{1}$ being the self- and junction capacitance, respectively. Although it is not essential in the subsequent discussion as long as the interaction range is finite, we assume for simplicity that $C_{1} / C_{0} \lesssim 1$, keeping only the on-site and the nearest-neighbor interactions between the charges. We also define charging energy scales $E_{0} \equiv e^{2} / 2 C_{0}, E_{1} \equiv e^{2} / 2 C_{1}$, and $E_{I} \equiv e^{2} / 2 C_{I}$, associated with the corresponding capacitances.

In the regime of concern in this paper, $C_{I} \gg C_{0}(\gtrsim$ $\left.C_{1}\right)$, i.e., $E_{I} \ll E_{0}\left(\lesssim E_{1}\right)$, the charging energy part of the Hamiltonian in Eq. (11) can be written conveniently as the sum $H_{C}=H_{C}^{+}+H_{C}^{-}$with each component defined to be

$$
\begin{aligned}
H_{C}^{+} \equiv & U_{0} \sum_{\mathbf{r}}\left[n_{+}(\mathbf{r})-2 n_{g}\right]^{2} \\
& +U_{1} \sum_{\mathbf{r}, \mu}\left[n_{+}(\mathbf{r})-2 n_{g}\right]\left[n_{+}\left(\mathbf{r}+\hat{\mathbf{e}}_{\mu}\right)-2 n_{g}\right] \\
H_{C}^{-} \equiv & V_{0} \sum_{\mathbf{r}}\left[n_{-}(\mathbf{r})\right]^{2}+V_{1} \sum_{\mathbf{r}, \mu} n_{-}(\mathbf{r}) n_{-}\left(\mathbf{r}+\hat{\mathbf{e}}_{\mu}\right)
\end{aligned}
$$

where we have defined new charge variables $n_{ \pm}(\mathbf{r}) \equiv$ $n_{1}(\mathbf{r}) \pm n_{2}(\mathbf{r})$ and the interaction strengths are given by $U_{0} \simeq 2 E_{0}, V_{0} \simeq E_{I}, U_{1} \simeq 4\left(C_{1} / C_{0}\right) E_{0}$, and $V_{1} \simeq$ $\left(C_{1} / C_{I}\right) E_{I}$. This representation of the charging energy part $H_{C}$ allows us to distinguish clearly the two interesting regions from each other: near the particle-hole symmetry line $n_{g}=0$ and near the maximal-frustration line $n_{g}=1 / 2$, as one can see from the energy spectra of $H_{C}$ displayed in Figs. 2 and 3 for the two regimes, respectively (recall that $U_{0} \gg V_{0}$ ). (Since the system is invariant with $n_{g} \rightarrow n_{g}+1$, we need to consider only the range $0 \leq n_{g}<1$ ). As pointed out for two coupled $1 \mathrm{D}$ arrays in Ref. 4, there follow the remarkable properties of the spectrum of $H_{C}$ in each regime: Near the maximalfrustration line, the charge configurations which do not satisfy the condition $n_{+}(\mathbf{r})=1$ (for all $\mathbf{r}$ ) have a huge excitation gap of the order of $E_{0}$. (Note that we are interested in the parameter regime $E_{I}, E_{J} \ll E_{0}$.) Furthermore, the ground states of $H_{C}$, separated from the excited states by the gap of the order of $E_{I}$, have twofold degeneracy for each $\mathbf{r}$, corresponding to $n_{-}(\mathbf{r})= \pm 1$. This degeneracy is lifted by the Josephson coupling term $H_{J}$ in Eq. (1) as $E_{J}$ is turned on. As a result, it is convenient in this case to work within the reduced Hilbert space $\mathcal{E}_{d}$, where $n_{+}(\mathbf{r})=1$ and $n_{-}(\mathbf{r})= \pm 1$ for each r. Near the particle-hole symmetry line, on the other hand, the low-energy charge configuration should satisfy the condition $n_{+}(\mathbf{r})=0$ for all $\mathbf{r}$. Unlike the former case, the ground state of $H_{C}$ is non-degenerate and forms a Mott insulator characterized by $n_{1}(\mathbf{r})=n_{2}(\mathbf{r})=0$ for all $\mathbf{r}$. As $E_{J}$ is turned on, the ground state of $H_{C}$ is mixed with the states with $n_{-}(\mathbf{r})= \pm 2$. Accordingly, the relevant reduced Hilbert space is given by $\mathcal{E}_{s}$, where $n_{+}(\mathbf{r})=0$ and $n_{-}(\mathbf{r})=0, \pm 2$ for all $\mathbf{r}$.

Accordingly, it is instructive to project the Hamiltonian in Eq. (1) onto $\mathcal{E}_{s}\left(\mathcal{E}_{d}\right)$ for $n_{g} \ll 1 / 4$ (for $\left|n_{g}-1 / 2\right| \ll$
$1 / 4)$; this results in the effective Hamiltonian, up to the second order in $E_{J} / E_{0}$,

$$
H_{e f f} \equiv P\left[H+H_{J} \frac{1-P}{E-H_{C}} H_{J}\right] P,
$$

where $P$ is the projection operator. 10 Explicit implementation of the projection near the particle-hole symmetry line can be achieved by first noting the correspondence between the charge picture of the original model and the pseudo-spin $(S=1)$ picture in the reduced Hilbert space $\mathcal{E}_{s}:$

$$
\begin{aligned}
S^{z}(\mathbf{r}) & \equiv P \frac{n_{1}(\mathbf{r})-n_{2}(\mathbf{r})}{2} P \\
S^{+}(\mathbf{r}) & \equiv \sqrt{2} P e^{-i \phi_{1}(\mathbf{r})}(1-P) e^{+i \phi_{2}(\mathbf{r})} P \\
S^{-}(\mathbf{r}) & \equiv \sqrt{2} P e^{-i \phi_{2}(\mathbf{r})}(1-P) e^{+i \phi_{1}(\mathbf{r})} P .
\end{aligned}
$$

In particular, the spin-flip operators $S^{+}$and $S^{-}$manifest the second-order cotunneling process of the particle-hole pairs via an intermediate virtual state, as depicted in Fig. $4(\mathrm{a})$, and mix the energy levels with unpaired particles or holes of energy $U_{0}$ and those with particle-hole pairs of energy $4 V_{0}$ (see Fig. 2). It then follows that the effective Hamiltonian in Eq. (5) takes the form

$$
\begin{aligned}
& H_{X X Z}^{S=1}=\frac{1}{2} \gamma_{1} J \sum_{\mathbf{r}}\left[S^{z}(\mathbf{r})\right]^{2} \\
& -\frac{1}{4} J \sum_{\mathbf{r}, \mu}\left\{S^{+}(\mathbf{r}) S^{-}\left(\mathbf{r}+\hat{\mathbf{e}}_{\mu}\right)+S^{-}(\mathbf{r}) S^{+}\left(\mathbf{r}+\hat{\mathbf{e}}_{\mu}\right)\right\}
\end{aligned}
$$

which describes the spin-1 2D XXZ antiferromagnet.11 Here the exchange interaction and the anisotropy ratio are given by

$$
J \simeq \frac{E_{J}^{2}}{4 E_{0}} \quad \text { and } \quad \gamma_{1} \simeq \frac{1}{K^{2}},
$$

respectively, with the dimensionless coupling constant $K \equiv \sqrt{E_{J}^{2} / 32 E_{I} E_{0}}$. Near the maximal-frustration line, on the other hand, the effective Hamiltonian reduces to that for a spin-1/2 2D XXZ antiferromagnet

$$
\begin{aligned}
& H_{X X Z}^{S=1 / 2}=\gamma_{\frac{1}{2}} J \sum_{\mathbf{r}, \mu} S^{z}(\mathbf{r}) S^{z}\left(\mathbf{r}+\hat{\mathbf{e}}_{\mu}\right) \\
& -\frac{1}{2} J \sum_{\mathbf{r}} \sum_{\mu}\left\{S^{+}(\mathbf{r}) S^{-}\left(\mathbf{r}+\hat{\mathbf{e}}_{\mu}\right)+S^{-}(\mathbf{r}) S^{+}\left(\mathbf{r}+\hat{\mathbf{e}}_{\mu}\right)\right\}
\end{aligned}
$$

with the exchange interaction and the anisotropic ratio given by

$$
J \simeq \frac{E_{J}^{2}}{4 E_{0}} \quad \text { and } \quad \gamma_{\frac{1}{2}} \simeq \frac{C_{1}}{2 C_{I}} \frac{1}{K^{2}},
$$

respectively. In this case, the definitions of the pseudospin operators in terms of the phase and charge operators are also different slightly from those in Eq. (6): 


$$
\begin{aligned}
& S^{z}(\mathbf{r}) \equiv P \frac{n_{1}(\mathbf{r})-n_{2}(\mathbf{r})}{2} P \\
& S^{+}(\mathbf{r}) \equiv P e^{-i \phi_{1}(\mathbf{r})}(1-P) e^{+i \phi_{2}(\mathbf{r})} P \\
& S^{-}(\mathbf{r}) \equiv P e^{-i \phi_{2}(\mathbf{r})}(1-P) e^{+i \phi_{1}(\mathbf{r})} P .
\end{aligned}
$$

Such spin-flip operators are associated with the cotunneling of the particle-void pairs as displayed in Fig. A(b).

In two dimensions, unlike the 1D case, neither of the two (spin-1 and spin-1/2) XXZ antiferromagnets described by Eqs. (7) and (9) allow exact solutions. The simple mean-field theory based on the Ginzburg-Landau approach ${ }^{12}$ indicates that the spin-1 XXZ antiferromagnet may exhibit a zero-temperature phase transition from the XY-like phase to the spin-1 Ising-like phase at $K \sim 1$ or $\gamma_{1} \sim 1$. In the charge picture, the XY-like phase corresponds to the superconducting (SC) state displaying ODLRO while the spin-1 Ising-like phase characterized by $S^{z}(\mathbf{r})=0$ describes the Mott insulator (MI) state with DLRO. On the other hand mean-field-like approaches 13 and numerical approaches14 to the spin- $1 / 2$ XXZ antiferromagnet suggest a zero-temperature phase transition from the XY-like phase to the spin-1/2 Ising-like phase with staggered magnetization at $K \sim \sqrt{C_{1} / 2 C_{I}}$ or $\gamma_{\frac{1}{2}} \sim 1$, corresponding to the SC state and the CDW state, respectively.

Therefore, for the present, the projection of the Hamiltonian to get the effective spin model does not provide us with direct information about the phase transitions. Remarkably, however, the spin models, given by Eqs. (7) and (9) in the two regimes, can be obtained from a single 2D quantum phase model (QPM), via appropriate projections. This strongly indicates that both regimes can be described by the Hamiltonian for the QPM:

$$
\begin{aligned}
H_{Q P M}= & 2 e^{2} \sum_{\mathbf{r}, \mathbf{r}^{\prime}}\left[n(\mathbf{r})-\tilde{n}_{g}\right] C^{-1}\left(\mathbf{r}, \mathbf{r}^{\prime}\right)\left[n\left(\mathbf{r}^{\prime}\right)-\tilde{n}_{g}\right] \\
& -\frac{E_{J}^{2}}{4 E_{0}} \sum_{\mathbf{r}, \mu} \cos \left[\phi(\mathbf{r})-\phi\left(\mathbf{r}+\hat{\mathbf{e}}_{\mu}\right)\right],
\end{aligned}
$$

where the effective Josephson-coupling energy $E_{J}^{2} / 4 E_{0}$ is much reduced compared with the original intra-array value $E_{J}$, and the effective capacitance matrix reads

$$
\begin{aligned}
C\left(\mathbf{r}, \mathbf{r}^{\prime}\right) & \equiv C_{I} \delta_{\mathbf{r r}^{\prime}} \\
& +\frac{C_{1}}{2} \sum_{\mu}\left[2 \delta_{\mathbf{r r}^{\prime}}-\delta_{\mathbf{r}, \mathbf{r}^{\prime}+\hat{\mathbf{e}}_{\mu}}-\delta_{\mathbf{r}, \mathbf{r}^{\prime}+\hat{\mathbf{e}}_{\mu}}\right] .
\end{aligned}
$$

Note that the self-capacitance is given by $C_{I}$ instead of the original value $C_{0}$ while the junction capacitance is given by $C_{1} / 2$. The value of charge frustration $\tilde{n}_{g}$ is related with that $\left(n_{g}\right)$ of the original model given by Eq. (1) in the following way: At the symmetry line $\left(n_{g}=0\right)$ and the maximal-frustration line $\left(n_{g}=1 / 2\right)$ of the original model, we have $\tilde{n}_{g}=n_{g}$. Near those lines, however, the value of $\tilde{n}_{g}$ is rather insensitive to that of $n_{g}$ : Namely, $\tilde{n}_{g}$ remains close to zero and to $1 / 2$ in the rather large ranges around $n_{g}=0$ and $1 / 2$, respectively, changing its value sharply near $n_{g} \approx 1 / 4$. Accordingly, the QPM in Eq. (12) is either near the symmetry line $\left(\tilde{n}_{g} \approx 0\right)$ or near the maximal-frustration line $\left(\tilde{n}_{g} \approx 1 / 2\right)$ except for the more or less narrow range around $n_{g}=1 / 4$.

The reduction of the above QPM to the spin-1 and the spin-1/2 XXZ models via appropriate projections can be recognized as follows: In the case $\tilde{n}_{g} \approx 0$, the charging energy reaches its minimum at $n(\mathbf{r})=0$. This ground state becomes mixed with the states $n(\mathbf{r})= \pm 1$, as the Josephson coupling is turned on. On the other hand, for $\tilde{n}_{g} \approx 1 / 2$, the minimum of the charging energy arises at $n(\mathbf{r})=0,1$, yielding two-fold degenerate ground states. These situations are essentially the same as those of the original model with charge frustration $n_{g}$. We thus project the QPM onto the spaces $\tilde{\mathcal{E}}_{s} \equiv\{n(\mathbf{r})=0, \pm 1\}$ and $\tilde{\mathcal{E}}_{d} \equiv\{n(\mathbf{r})=0,1\}$ with the psuedo-spin operators redefined as

$$
\begin{aligned}
S^{z}(\mathbf{r}) & \equiv P n(\mathbf{r}) P \\
S^{+}(\mathbf{r}) & \equiv \sqrt{2} P e^{-i \phi(\mathbf{r})} P \\
S^{-}(\mathbf{r}) & \equiv \sqrt{2} P e^{i \phi(\mathbf{r})} P
\end{aligned}
$$

in space $\tilde{\mathcal{E}}_{s}$ and

$$
\begin{aligned}
S^{z}(\mathbf{r}) & \equiv P n(\mathbf{r}) P-1 / 2 \\
S^{+}(\mathbf{r}) & \equiv P e^{-i \phi(\mathbf{r})} P \\
S^{-}(\mathbf{r}) & \equiv P e^{i \phi(\mathbf{r})} P
\end{aligned}
$$

in space $\tilde{\mathcal{E}}_{d}$; these projections reproduce, in the zeroth order of $E_{J} / E_{I}$, both the spin- 1 and the spin- $1 / 2 \mathrm{XXZ}$ models in Eqs. (7) and (9) for $n_{g} \ll 1 / 4$ and $\left|n_{g}-1 / 2\right| \ll$ $1 / 4$, respectively.

As we proceed to higher orders, the projection of the single-layer QPM in Eq. (12) in general yields the coefficients of the $n$ th-order terms $\left(E_{0} / E_{I}\right)^{(n-1)}$ times larger than those in the projection of the original model in Eq. (11). In spite of such discrepancy in numerical coefficients, the two projections (of the original model and of the single-layer QPM) onto their own spin models should bring about quite similar structures. For example, mixing of the energy levels in $\mathcal{E}_{s}$ with those satisfying $n_{-}(\mathbf{r})= \pm 4, \pm 6, \cdots$ (but still keeping $n_{+}(\mathbf{r})=0$ ) always occurs via the virtual states with energies of the order of $E_{0}$. Consequently, at least in the two regimes of concern here, it is not irrelevant to consider the single-layer QPM in Eq. (12) as an effective model for the original system. Quite naturally, the deviation of the QPM in Eq. (12) from the original model increases with $E_{J}$.

The 2D QPM has been studied extensively in recent years (see, e.g., Ref. (8). Remarkably, for $\left|n_{g}-1 / 2\right| \ll 1 / 4$, it was suggested that there may exist an unusual SS phase with both the DLRO and ODLRO, i.e., the coexistence of the crystalline charge ordering together with superconductivity. The existence of the SS phase conflicts with the prediction of a direct transition from the CDW to the SC based on the spin-1/2 XXZ model in Eq. (9), 
but such conflict also appears when one simply trun cates the effects of higher energy levels in the QPM.8 These arguments finally yield the schematic phase diagram shown in Fig. 同, where the thick solid lines represent the phase boundaries of the SI transitions, separating the SC from the MI (near the symmetry line) or from the CDW (near the maximal-frustration line depicted by the dashed-dotted line). Note that these boundaries (near the two lines) change rather gradually as $n_{g}$ is varied, which reflects that near the two lines the effective charge frustration $\tilde{n}_{g}$ in the QPM is insensitive to the original charge frustration $n_{g}$. The dashed lines in Fig. 5 represent the somewhat speculative boundaries discussed above; here the region occupied by the SS phase might be small because in the QPM the self-capacitance is much larger than the junction capacitance $\left(C_{I} \gg C_{1} / 2\right)$.

In conclusion, we have investigated the quantum phase transitions in two capacitively coupled two-dimensional Josephson-junction arrays with charge frustration. The system has been mapped into the $S=1$ and the $S=1 / 2$ anisotropic XXZ antiferromagnets near the particle-hole symmetry line and the maximal-frustration line, respectively. We have then argued that the two spin models in effect can be incorporated into a single quantum phase model. Based on the resulting model, it has been suggested that near the maximal frustration line the system may exhibit a quantum phase transition from the chargedensity wave to the super-solid phase, displaying both diagonal and off-diagonal long-range order.

This work was supported in part by the SNU Research Fund, by the Korea Research Foundation, and by the Korea Science and Engineering Foundation.

* Present address: Department of Physics and Astronomy, University of Basel, 4056 Basel, Switzerland.

${ }^{1}$ D. V. Averin and Y. V. Nazarov, in Single Charge Tunneling: Coulomb Blockade Phenomena in Nanostructures, edited by H. Grabert and M. H. Devoret (Plenum Press, New York, 1992), p. 217.

${ }^{2}$ D. V. Averin, A. N. Korotkov, and Y. V. Nazarov, Phys. Rev. Lett. 66, 2818 (1991).

${ }^{3}$ M. Matters, J. J. Versluys, and J. E. Mooij, Phys. Rev. Lett. 78, 2469 (1997).

${ }^{4}$ M.-S. Choi, M. Y. Choi, T. Choi, and S.-I. Lee, Phys. Rev. Lett. 81, 4240 (1998).

${ }^{5}$ M.-S. Choi, M. Y. Choi, and S.-I. Lee, preprint condmat/9802237.).

${ }^{6}$ M.-S. Choi, J. Phys.: Condens. Matter 10, 9783 (1998).

${ }^{7}$ Ya. M. Blanter and G. Schön, Phys. Rev. B 53, 14534 (1996); Ya. M. Blanter, R. Fazio, and G. Schön, Nucl. Phys. B S58, 79 (1997); J. V. José, J. Stat. Phys. 93, 943 (1998).

${ }^{8}$ A. van Otterlo and K.-H. Wagenblast, Phys. Rev. Lett. 72, 3598 (1994); A. van Otterlo, K.-H. Wagenblast, R. Baltin, C. Bruder, R. Fazio, and G. Schön, Phys. Rev. B 52, 16176
(1995).

${ }^{9}$ G. Blatter, M. V. Feigel'man, V. B. Geshkenbein, A. I. Larkin, and V. M. Vinokur, Rev. Mod. Phys. 66, 1125 (1994).

${ }^{10}$ C. Bruder, R. Fazio, and G. Schön, Phys. Rev. B 47, 342 (1993); L. I. Glazman and A. I. Larkin, Phys. Rev. Lett. 79, 3736 (1997).

11 The sign of the second term in Eq. (7) is unimportant on a biparticle lattice; see, e.g., A. Auerbach, Interacting Electrons and Quantum Magnetism (Springer-Verlag, Berlin/Heidelberg, 1994).

12 M.-S. Choi and M. Y. Choi, unpublished.

${ }^{13}$ D. J. Bukman and J. M. J. van Leeuwen, J. Phys. A 25, 4285 (1992); M.-R. Li, Y.-J. Wang, and C.-D. Gong, Z. Phys. B 102, 129 (1992).

${ }^{14}$ T. Barnes, D. Kotchan, and E.S. Swanson, Phys. Rev. B 39, 4357 (1989); M. Kikuchi, Y. Okabe, and S. Miyashita, J. Phys. Soc. Jpn. 59, 492 (1990). 


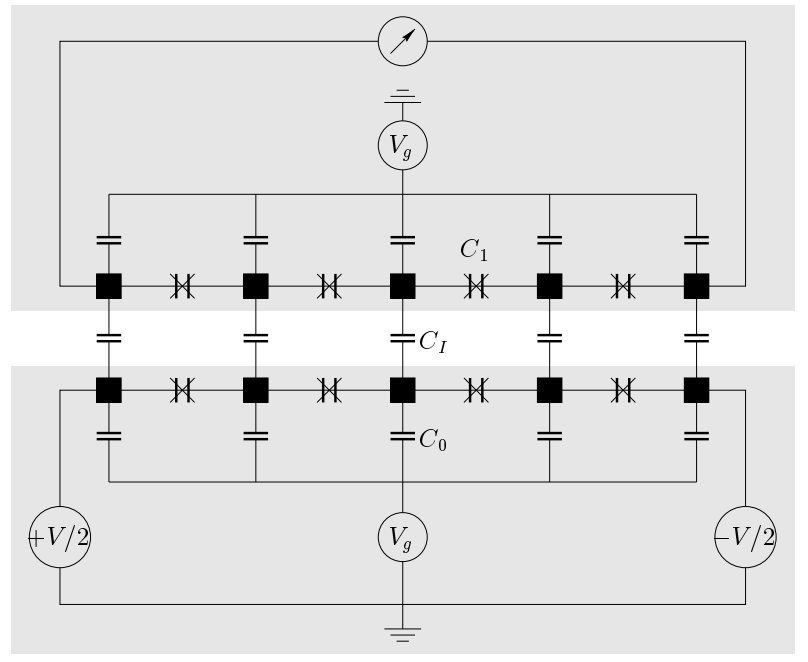

FIG. 1. Schematic diagram of the two coupled 2D arrays. Each of the upper and lower arrays represents a 2D array composing the system.

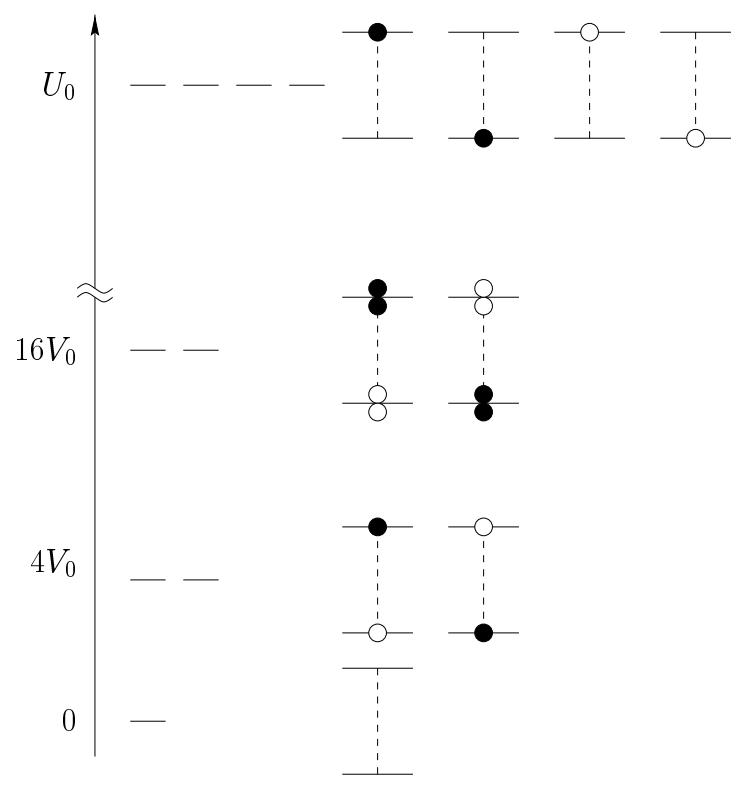

FIG. 2. Energy levels of $H_{C}$ in Eqs. (4) and corresponding charge configurations near the particle-hole symmetry line. Filled and empty circles denote particles and holes, respectively; paired (upper and lower) solid lines represent the two coupled arrays, the couplings between which are illustrated by the dashed lines. The low-lying energy levels satisfying $n_{+}(\mathbf{r})=0$ are well separated by a large amount of energy (of the order of $\left.E_{0}\right)$ from those with $n_{+}(\mathbf{r}) \neq 0$.

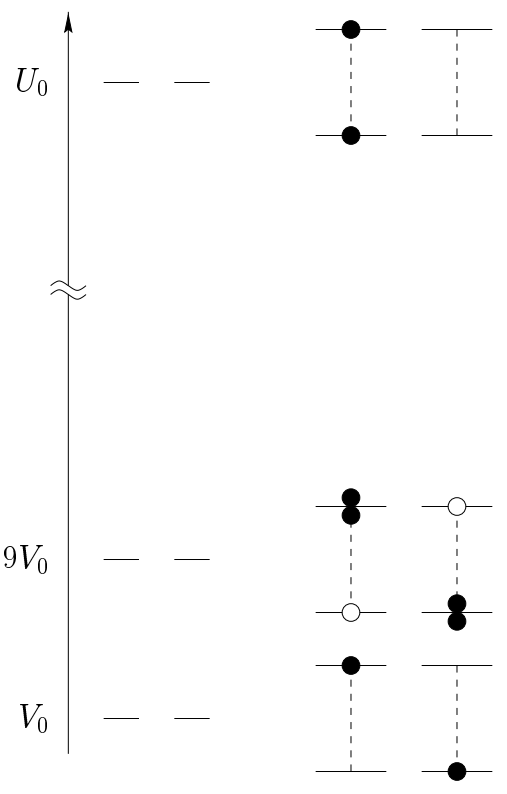

FIG. 3. Energy levels and corresponding charge configurations near the maximal-frustration line. It should be noticed that the ground state is two-fold degenerate per site.

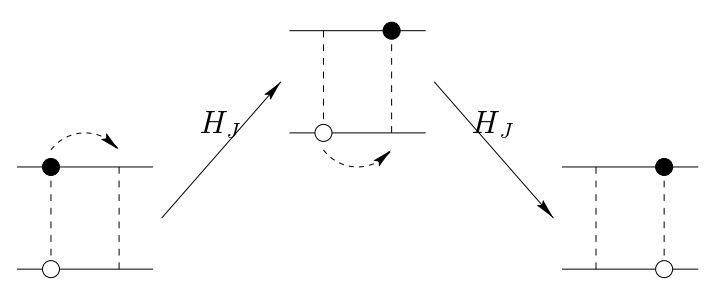

(a)

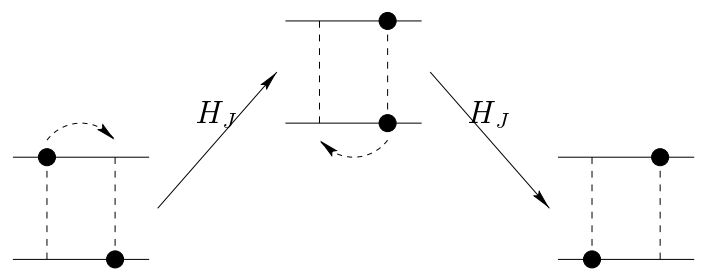

(b)

FIG. 4. Typical cotunneling processes relevant (a) near the particle-hole symmetry line and (b) near the maximal-frustration line. The intermediate virtual state costs an energy of the order of $E_{0}$. 


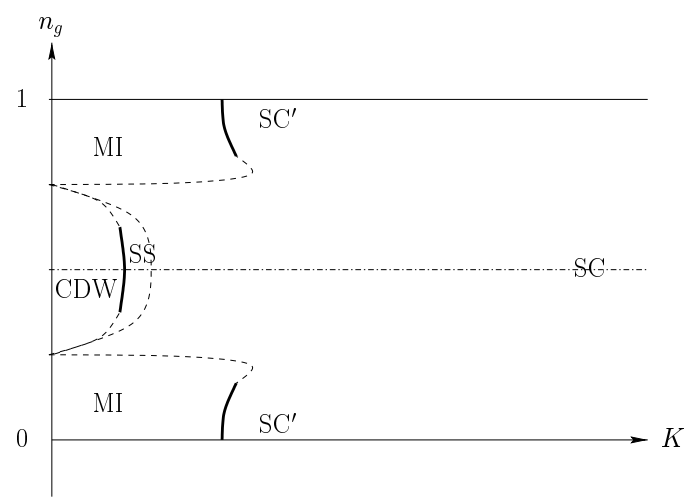

FIG. 5. Schematic phase diagram of the system. The superconducting $\mathrm{SC}^{\prime}$ phase is distinguished from the $\mathrm{SC}$ phase in that the underlying transport mechanism is the cotunneling process instead of the single-charge transport. 